\title{
Robust Proactive Project Scheduling Model for the Stochastic Discrete Time/Cost Trade-Off Problem
}

\author{
Hongbo Li, ${ }^{1} \mathrm{Zhe} \mathrm{Xu}^{2}{ }^{2} \mathrm{Li}$ Xiong, ${ }^{1}$ and Yinbin Liu ${ }^{1}$ \\ ${ }^{1}$ School of Management, Shanghai University, 99 Shangda Road, Shanghai 200444, China \\ ${ }^{2}$ School of Economics and Management, Beihang University, 37 Xueyuan Road, Beijing 100191, China
}

Correspondence should be addressed to Yinbin Liu; yinbinliu@126.com

Received 21 August 2014; Accepted 4 November 2014

Academic Editor: Hua Gong

Copyright (C) 2015 Hongbo Li et al. This is an open access article distributed under the Creative Commons Attribution License, which permits unrestricted use, distribution, and reproduction in any medium, provided the original work is properly cited.

\begin{abstract}
We study the project budget version of the stochastic discrete time/cost trade-off problem (SDTCTP-B) from the viewpoint of the robustness in the scheduling. Given the project budget and a set of activity execution modes, each with uncertain activity time and cost, the objective of the SDTCTP-B is to minimize the expected project makespan by determining each activity's mode and starting time. By modeling the activity time and cost using interval numbers, we propose a proactive project scheduling model for the SDTCTP-B based on robust optimization theory. Our model can generate robust baseline schedules that enable a freely adjustable level of robustness. We convert our model into its robust counterpart using a form of the mixed-integer programming model. Extensive experiments are performed on a large number of randomly generated networks to validate our model. Moreover, simulation is used to investigate the trade-off between the advantages and the disadvantages of our robust proactive project scheduling model.
\end{abstract}

\section{Introduction}

In project management, the project duration can usually be shortened by allocating more resources to critical activities. The number of resources tends to be discrete, such as the numbers of workers and machines. These resources are usually treated as nonrenewable resources and measured by capital (or cost), resulting in the discrete time/cost trade-off problem (DTCTP) [1]; Harvey and Patterson [2] and Hindelang and Muth [3] first proposed the DTCTP, which is a special case of the multimode resource-constrained project scheduling problem [4].

In the deterministic DTCTP, each activity has multiple execution modes that are characterized by specific time and cost combinations. In terms of the types of the objective function, the DTCTP can be divided into three versions: the deadline problem (DTCTP-D), the budget problem (DTCTP-B), and the time/cost trade-off curve problem (DTCTP-C). In the DTCTP-D, given a set of modes and a project deadline, the objective is to minimize the total project cost by specifying for each activity an execution mode. In the DTCTP-B, a project budget is given and the objective is to determine the modes that minimize the project makespan. In the DTCTP-C, the goal is to determine the Pareto curve that minimizes the project cost and makespan simultaneously.

Once the mode of each activity is determined, we can determine the baseline schedule $S^{B}=\left(s_{1}, s_{2}, \ldots, s_{n}\right)$ by calculating the earliest start time of each activity in accordance with the critical path method (CPM).

However, during project execution, due to considerable uncertainties, the optimal baseline schedule which is obtained based on a deterministic environment and complete information may deviate from our expectations or even become unfeasible. Possible sources of uncertainties may be a shortage of machineries, a delayed delivery of materials, the absence of workers, fluctuations in the exchange rates, and so forth $[5,6]$. As a consequence, issues such as schedule delays and/or budget overruns may occur and project time and cost objectives will be threatened. Therefore, there is a pressing need for new procedures for the DTCTP under uncertainties to obtain project schedules which are insensitive to disruption.

Recent studies have paid more attention to the stochastic DTCTP (SDTCTP), which accounts for uncertainties by 
treating the time and cost of activities as stochastic variables, with the objective of optimizing the expected project performance. As early researchers in the field, Gutjahr et al. [7] presented a stochastic branch-and-bound procedure to solve the DTCTP deadline problem based on the assumption that the times of each activity are mutually independent random variables. Laslo [8] used the fractal method to construct time/cost curves for a single activity of stochastic duration. Cohen et al. [9] implemented a robust optimization to solve the time/cost trade-off problem. Ke et al. [10] used chanceconstrained programming and dependent-chance programming to model the stochastic DTCTP; the authors designed an intelligent algorithm to search the quasi-optimal schedules while balancing the project duration and cost. Klerides and Hadjiconstantinou [11] proposed a path-based two-stage stochastic integer programming approach to decide how and when to execute each activity to minimize the project duration or cost using realized activity durations. Ma et al. [12] studied the stochastic time-cost-quality trade-off problem where the activity durations are uncertain and developed a hybrid genetic algorithm.

However, the above-mentioned research papers primarily focused on optimizing the system performance in an average sense and these prior approaches cannot guarantee the performance of the baseline schedule during a single project execution. Therefore, determining a robust baseline schedule under uncertainty is increasingly attracting the attention of scholars. To achieve a robust baseline schedule, the use of robust optimization is a natural choice. Robust optimization can determine a solution with certain robustness by optimizing the worst-case performance of the system. Although robust optimization has been used to solve some classic project scheduling problems $[13,14]$, little effort has been applied to the study of the SDTCTP. To the best of our knowledge, Hazir et al. [6] is the only research article that combines the SDTCTP and the robust optimization approach. Hazir et al. propose three robust optimization models, in which cost uncertainty is modeled via intervals for the SDTCTP deadline problem. The aim of their model is to minimize the effect of unexpected events on project performance. The limitations of their models are that the activity cost is still assumed to be deterministic and that only the parameters in the objective function are subject to uncertainty (i.e., the parameters in the constraints are deterministic).

To the best of our knowledge, addressing both time- and cost-uncertainty and applying robust optimization in solving the SDTCTP-B have not been taken into account in both the project scheduling and the robust optimization literature. The contributions of this paper are as follows.

(1) We proposed a proactive scheduling model for the SDTCTP budget problem (SDTCTP-B) based on robust optimization theory. Our model uses interval numbers to model the uncertain time and cost of the activities that can follow any type of probability distribution. The objective of our model is to generate a stable baseline schedule that can account for some of the uncertainties during project execution to ensure, to the extent possible, that each activity begins at their respective planned start time.

(2) We conducted a detailed experimental analysis for our proposed model. We used experimental design to randomly generate a large number of instances to validate our model. In addition, robust optimization improves the schedule stability at the cost of prolonging the project duration. Therefore, we used simulation to investigate the trade-off between the advantages and the disadvantages of robust optimization. Specifically, we analyzed the impact of the number of activities, the network order strength, and the number of modes on the schedule stability by using discrete systems simulation.

This paper is organized as follows. Section 2 provides a description of the SDTCTP. In Section 3, we use interval numbers to model the uncertain parameters and present a proactive model to solve the SDTCTP-B based on robust optimization. Considering the nonlinear characteristics of the proposed model, we converted our model into its robust counterpart, which has the form of a mixed integer linear programming model, and used the branch-and-cut algorithm to solve the model. In Section 4, we present the experimental results. Finally, Section 5 concludes the paper.

\section{Problem Description}

The stochastic discrete time/cost trade-off problem can be described as follows. A project network $G=(N, A)$ is represented in the activity-on-node format, where the set of nodes $N$ denotes the activities $N=\{1,2, \ldots, n\}$ and the set of directed arcs $A$ represents the finish-start, zero-lag precedence relations $A \subseteq N \times N$. The nodes are topologically numbered from the single-start node 1 to the single-terminal node $n, n=|N|$, where nodes 1 and $n$ represent two dummy activities. The duration and cost of activity $i$ are random variables, denoted as $\widetilde{d}_{i m}$ and $\widetilde{c}_{i m}$, respectively. For activity $i, M_{i}$ represents the set of its modes. Each activity $i(i=1, \ldots, n)$ has $\left|M_{i}\right|$ modes, which are characterized by a duration-cost pair $\left(\widetilde{d}_{i m}, \widetilde{c}_{i m}\right), m=1, \ldots,\left|M_{i}\right|$. The duration $\widetilde{d}_{i m}$ of an activity $i \in N$ is a discrete, nonincreasing function of the amount of the single nonrenewable resource $\left(\widetilde{c}_{i m}\right)$ committed to it; that is, if $k<l\left(k, l \in M_{i}\right)$, then, for the expected duration and cost, we have $E\left(d_{i l}\right)<E\left(d_{i k}\right)$ and $E\left(c_{i l}\right)>E\left(c_{i k}\right)$. We assume that the dummy activities 1 and $n$ have only one execution mode of zero duration/cost.

Given the project budget $R$, the objective of the SDTCTP$\mathrm{B}$ is to minimize the expected project makespan by assigning a mode $m_{j}\left(j=1, \ldots, M_{i}\right)$ to each activity and determining the start time $s_{i}$ of each activity. If we substitute the random duration and cost of the activities by their most likely values $d_{i m}$ and $c_{i m}$, respectively, for the above problem, the SDTCTP will become the deterministic DTCTP. For DTCTP-B, we have the following integer programming model:

$$
\begin{array}{ll}
\text { minimize } & s_{n} \\
\text { subject to } & \sum_{m \in M_{i}} x_{i m}=1 \quad \forall i \in N
\end{array}
$$




$$
\begin{aligned}
& s_{i}+\sum_{m \in M_{i}} d_{i m} x_{i m} \leq s_{j} \quad \forall(i, j) \in A \\
& \sum_{i \in N} \sum_{m \in M_{i}} c_{i m} x_{i m} \leq R \\
& s_{i} \geq 0 \quad \forall i \in N \\
& x_{i m} \in\{0,1\} \quad \forall m \in M_{j}, \forall i \in N,
\end{aligned}
$$

where $s_{i}$ and $x_{i m}$ are decision variables. $x_{i m}$ are $0-1$ variables that determine whether a mode of an activity is selected. The objective function (1) minimizes the start time of the dummy end node $n$, which is equivalent to minimizing the project makespan. Equation (2) ensures that for each activity only one execution mode is selected. Equation (3) defines the precedence relationship constraints for the activities. Equation (4) ensures that the total project cost does not exceed the budget $R$. Equation (5) ensures that the start time of each activity is nonnegative. The budget problem of DTCTP is strongly NP-hard $[1,15,16]$.

Faced with the uncertainty in the duration and cost of each activity, the baseline schedule generated by the above deterministic model is not expected to be executed as determined, thereby resulting in failure to achieve the desired project objective. When the uncertainty is considered, we notice that the uncertain parameters mainly affect constraints (3) and (4). Therefore, in the following section, we model the uncertain parameters as interval numbers and develop a proactive scheduling model for the SDTCTP-B based on robust optimization, thereby obtaining a robust baseline schedule.

\section{The Proactive Scheduling Model for SDTCTP-B}

3.1. Modeling Uncertain Parameters as Interval Numbers. In practice, it is usually easier for decision-makers to estimate the range and the most likely value of the duration and cost of activities rather than their probability distribution. Therefore, we use interval numbers to model the uncertain duration and cost of the activity. For mode $m$, let $d_{i m}$ and $c_{i m}$ be the most likely value of the activity duration $\widetilde{d}_{i m}$ and cost $\widetilde{c}_{i m}$, respectively; $d_{i m}$ and $c_{i m}$ take a value in the interval $\left[\underline{d}_{i m}, \bar{d}_{i m}\right]$ and $\left[\underline{c}_{i m}, \bar{c}_{i m}\right]$, respectively (i.e., $\underline{d}_{i m}<d_{i m}<\bar{d}_{i m}, \underline{c}_{i m}<$ $\left.c_{i m}<\bar{c}_{i m}\right)$. We define the maximum deviation of the activity duration and cost as $\widehat{d}_{i m}=\max \left\{d_{i m}-\underline{d}_{i m}, \bar{d}_{i m}-d_{i m}\right\}$ and $\widehat{c}_{i m}=\max \left\{c_{i m}-\underline{c}_{i m}, \bar{c}_{i m}-c_{i m}\right\}$, respectively, which represent the maximum difference between the planned activity duration and cost and the actual activity duration and cost that can be tolerated by the decision-makers.

3.2. The Proactive Scheduling Model. For each activity $i$, we introduce a parameter $\Gamma_{i}$, which is not necessarily an integer, that takes on a value in the interval $\left[0,\left|M_{i}\right|\right] . \Gamma_{i}$ is used to adjust the robustness of our model (i.e., the level of conservatism of the solutions). For each activity $i$, we assume that $\left\lfloor\Gamma_{i}\right\rfloor$ modes take on the values at their upper bounds of $\bar{d}_{i m}, \bar{c}_{i m}$, the value for one mode can deviate $\left(\Gamma_{i}-\left\lfloor\Gamma_{i}\right\rfloor\right) \widehat{d}_{i m}$ and $\left(\Gamma_{i}-\left\lfloor\Gamma_{i}\right\rfloor\right) \widehat{c}_{i m}$, and the remaining $\left|M_{i}\right|-\left\lfloor\Gamma_{i}\right\rfloor$ modes are set to their most likely values of $d_{i m}, c_{i m}$.

Our proactive scheduling model for SDTCTP-B based on robust optimization $[17,18]$ is as follows:

$$
\begin{aligned}
& \text { (Model 1) minimize } s_{n} \\
& \text { subject to } s_{i}+\sum_{m \in M_{i}} d_{i m} x_{i m} \\
& +\max _{\left\{S_{i} \cup\left\{t_{i}\right\}\left|S_{i} \subseteq M_{i},\right| S_{i} \mid=\left\lfloor\Gamma_{i}\right\rfloor, t_{i} \in M_{i} \backslash S_{i}\right\}}\left\{\sum_{k \in S_{i}} \widehat{d}_{i k} x_{i k}+\left(\Gamma_{i}-\left\lfloor\Gamma_{i}\right\rfloor\right) \widehat{d}_{i t_{i}} x_{i t_{i}}\right\} \leq s_{j} \quad \forall(i, j) \in A \\
& \sum_{i \in N} \sum_{m \in M_{i}} c_{i m} x_{i m}+\sum_{i \in N} \max _{\left\{S_{i} \cup\left\{t_{i}\right\}\left|S_{i} \subseteq M_{i},\right| S_{i} \mid=\left\lfloor\Gamma_{i}\right\rfloor, t_{i} \in M_{i} \backslash S_{i}\right\}}\left\{\sum_{k \in S_{i}} \widehat{c}_{i k} x_{i k}+\left(\Gamma_{i}-\left\lfloor\Gamma_{i}\right\rfloor\right) \widehat{c}_{i t_{i}} x_{i t_{i}}\right\} \leq R
\end{aligned}
$$

$(2),(5),(6)$.

The above model introduces the uncertain parameters into the deterministic DTCTP model and is able to generate robust solutions. $S_{i}$ is a subset of $M_{i}$, and the modes belonging to $S_{i}$ take on the worst case values. The cardinality of $S_{i}$ is determined by $\Gamma_{i}:\left|S_{i}\right|=\left\lfloor\Gamma_{i}\right\rfloor$. In addition, the objective function (7) is a deterministic function rather than an expectation function. Therefore, the time-consuming expectation calculation is avoided.
Our model has two primary advantages. The first advantage is that the robustness level of the obtained schedule can be freely adjusted. The greater the value of the parameter $\Gamma_{i}$, the higher the level of robustness is. If $\Gamma_{i}=0$, the model will become the deterministic DTCTP model. The second advantage is that, although the proposed model is nonlinear, it can be easily reformulated as an equivalent linear mixed-integer programming (MIP) model according to 
the robust optimization theory $[17,18]$. To obtain the equivalent MIP model, we first let $g\left(\mathbf{x}, \Gamma_{i}\right)=$ $\max _{\left\{S_{i} \cup\left\{t_{i}\right\}\left|S_{i} \subseteq M_{i},\right| S_{i} \mid=\left\lfloor\Gamma_{i}\right\rfloor, t_{i} \in M_{i} \backslash S_{i}\right\}}\left\{\sum_{k \in s_{i}} \widehat{d}_{i k} x_{i k}+\left(\Gamma_{i}-\left\lfloor\Gamma_{i}\right\rfloor\right) \widehat{d}_{i t_{i}} x_{i t}\right\}$. $g\left(\mathbf{x}, \Gamma_{i}\right)$ means that we need to determine a subset $\left\{S_{i} \cup\right.$ $\left.\left\{t_{i}\right\} \quad\left|S_{i} \subseteq M_{i},\right| S_{i} \mid=\left\lfloor\Gamma_{i}\right\rfloor, t_{i} \in M_{i} \backslash S_{i}\right\}$ that includes $\left\lfloor\Gamma_{i}\right\rfloor+1$ elements, such that $\sum_{k \in s_{i}} \widehat{d}_{i k} x_{i k}+\left(\Gamma_{i}-\left\lfloor\Gamma_{i}\right\rfloor\right) \widehat{d}_{i t_{i}} x_{i t}$ is maximized. Therefore, we introduce $\left\lfloor\Gamma_{i}\right\rfloor+1$ decision variables $z_{i k}, 0 \leq z_{i k} \leq 1, k \in M_{i}$. Given a vector $\mathbf{x}^{*}, g\left(\mathbf{x}^{*}, \Gamma_{i}\right)$ equals the objective function value of the following linear programming:

$$
\begin{aligned}
g\left(\mathbf{x}^{*}, \Gamma_{i}\right)=\operatorname{maximize} & \sum_{k \in M_{i}} \widehat{d}_{i k} x_{i k}^{*} z_{i k} \\
\text { subject to } & \sum_{k \in M_{i}} z_{i k} \leq \Gamma_{i} \\
& 0 \leq z_{i k} \leq 1 \quad \forall k \in M_{i} .
\end{aligned}
$$

The constraints of problem (10) ensue that the resulting optimal objective function value is equivalent to $g\left(\mathbf{x}^{*}, \Gamma_{i}\right)$. It is clear that the linear programming problem (10) has an optimal solution with $\left\lfloor\Gamma_{i}\right\rfloor$ of decision variables taking on the value of 1 and the remaining $\Gamma_{i}-\left\lfloor\Gamma_{i}\right\rfloor$ taking on the value 0 .

The dual of problem (10) is

$$
\begin{array}{ll}
\operatorname{minimize} & \Gamma_{i} z_{i}+\sum_{k \in M_{i}} p_{i k} \\
\text { subject to } & z_{i}+p_{i k} \geq \widehat{d}_{i k} x_{i k}^{*} \quad \forall k \in M_{i}, \forall i \in N \\
& p_{i k} \geq 0 \quad \forall k \in M_{i} \\
& z_{i} \geq 0 \quad \forall i \in N,
\end{array}
$$

where $z_{i}$ and $p_{i k}$ are dual variables. According to strong duality, because problem (10) has an optimal solution, then problem (11) also has an optimal solution, and their optimal values are the same. In addition, $g\left(\mathbf{x}^{*}, \Gamma_{i}\right)$ equals the objective function value of problem (11).

Similarly, the "max" part of (9) can be converted to the following linear programming model:

$$
\begin{array}{ll}
\operatorname{minimize} & \Gamma_{i} y_{i}+\sum_{k \in M_{i}} q_{i k} \\
\text { subject to } & y_{i}+q_{i k} \geq \widehat{c}_{i k} x_{i k}^{*} \quad \forall k \in M_{i}, \quad \forall i \in N \\
& q_{i k} \geq 0 \quad \forall k \in M_{i} \\
& y_{i} \geq 0 \quad \forall i \in N .
\end{array}
$$

Then we can obtain the equivalent mixed-integer linear optimization model of Model 1 by substituting (11) and (12) into it:

$$
\begin{array}{ll}
\text { minimize } & s_{n} \\
\text { subject to } & \sum_{m \in M_{i}} x_{i m}=1 \quad \forall i \in N \\
& s_{i}+\sum_{m \in M_{i}} d_{i m} x_{i m}+\Gamma_{i} z_{i}+\sum_{k \in M_{i}} p_{i k} \leq s_{j} \\
& \forall(i, j) \in A \\
& z_{i}+p_{i k} \geq \widehat{d}_{i k} x_{i k} \quad \forall k \in M_{i}, \quad \forall i \in N
\end{array}
$$

$$
\begin{aligned}
& \sum_{i \in N} \sum_{m \in M_{i}} c_{i m} x_{i m}+\sum_{i \in N}\left(\Gamma_{i} y_{i}+\sum_{k \in M_{i}} q_{i k}\right) \leq R \\
& y_{i}+q_{i k} \geq \widehat{c}_{i k} x_{i k} \quad \forall k \in M_{i}, \forall i \in N \\
& p_{i k} \geq 0 \quad \forall k \in M_{i} \\
& z_{i} \geq 0 \quad \forall i \in N \\
& q_{i k} \geq 0 \quad \forall k \in M_{i} \\
& y_{i} \geq 0 \quad \forall i \in N \\
& s_{i} \geq 0 \quad \forall i \in N \\
& x_{i m} \in\{0,1\} \quad \forall m \in M_{j}, \quad \forall i \in N .
\end{aligned}
$$

3.3. Example. We use an example project network in Figure 1 to illustrate our model. Activities 0 and 5 are dummy activities. Activities 1 and 2 have only one mode. Activities 3 and 4 have two modes. We assume that the project budget is $R=75$.

Table 1 lists all the possible mode combinations for the different values of $\Gamma$. The first column lists the $\Gamma$ values. The second and third columns indicate the selected modes for activities 3 and 4. The column labeled "Total cost" shows the total project cost given the chosen activities modes. The column labeled "Makespan" represents the objective function value (i.e., the project makespan). The column labeled "Feasible" reveals whether the given mode combination is feasible, given the budget constraint of 75 .

As shown in Table 1 , when $\Gamma=0$, all the modes use their most likely values and we have two solutions with the makespan of 13 . When $\Gamma=1$, the model is a robust model and we have three solutions with the makespan of 13. Among the three solutions, two of them are the same as the case of $\Gamma=1$ and the third one (the corresponding row in Table 1 is bold) can be regarded as a robust solution. For the robust solution, because the duration of activity 4 takes on the worstcase value, this will result in a lower probability of delaying the project if activity 4 is disrupted by uncertain factors. When $\Gamma=2$, all modes use their worst-case values and we have one solution with the makespan of 15 . The maximum $\Gamma$ makes the project schedule most robust, at the price of accepting a larger project makespan.

\section{Computational Experiments}

We have randomly generated a large number of problem instances to validate our model. Section 4.1 describes how to generate these instances. Next, the optimization results with different parameters settings are reported in Section 4.2. Section 4.3 describes the use of simulation to investigate the impact of different project network parameters on the schedule stability. The experiments were conducted in Matlab version R2010b and run on an Intel Core i5 $2.40 \mathrm{GHz}$ portable computer under Windows 7. We used Matlab to call the builtin branch-and-cut algorithm of CPLEX 12.4 to solve the MIP. 


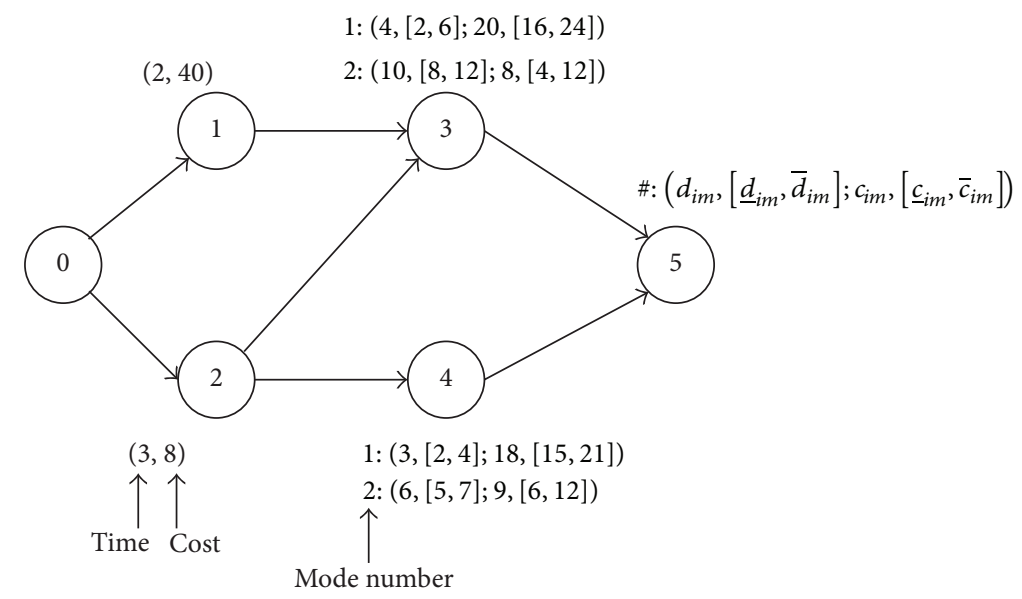

FIGURE 1: The example project network.

TABLE 1: Computations for the example problem.

\begin{tabular}{|c|c|c|c|c|c|}
\hline \multirow{2}{*}{$\Gamma$} & \multicolumn{2}{|c|}{ Mode } & \multirow{2}{*}{ Total cost } & \multirow{2}{*}{ Makespan } & \multirow{2}{*}{ Feasible } \\
\hline & Activity 3 & Activity 4 & & & \\
\hline \multirow{4}{*}{0} & 1 & 1 & 86 & 7 & No \\
\hline & 1 & 2 & 77 & 9 & No \\
\hline & 2 & 1 & 74 & 13 & Yes \\
\hline & 2 & 2 & 65 & 13 & Yes \\
\hline \multirow{16}{*}{1} & 1 & 1 & 86 & 7 & No \\
\hline & 1 & $1^{*}$ & 89 & 7 & No \\
\hline & 1 & 2 & 77 & 9 & No \\
\hline & 1 & $2^{*}$ & 80 & 10 & No \\
\hline & $1^{*}$ & 1 & 90 & 9 & No \\
\hline & $1^{*}$ & $1^{*}$ & 93 & 9 & No \\
\hline & $1^{*}$ & 2 & 81 & 9 & No \\
\hline & $1^{*}$ & $2^{*}$ & 84 & 10 & No \\
\hline & 2 & 1 & 74 & 13 & Yes \\
\hline & 2 & $1^{*}$ & 77 & 13 & No \\
\hline & 2 & 2 & 65 & 13 & Yes \\
\hline & 2 & $2^{*}$ & 68 & 13 & Yes \\
\hline & $2^{*}$ & 1 & 78 & 15 & No \\
\hline & $2^{*}$ & $1^{*}$ & 81 & 15 & No \\
\hline & $2^{*}$ & 2 & 69 & 15 & Yes \\
\hline & $2^{*}$ & $2^{*}$ & 72 & 15 & Yes \\
\hline \multirow{4}{*}{2} & $1^{*}$ & $1^{*}$ & 93 & 9 & No \\
\hline & $1^{*}$ & $2^{*}$ & 84 & 10 & No \\
\hline & $2^{*}$ & $1^{*}$ & 81 & 15 & No \\
\hline & $2^{*}$ & $2^{*}$ & 72 & 15 & Yes \\
\hline
\end{tabular}

${ }^{*}$ This mode uses the worst-case value.

4.1. Data Generation and Settings. RanGen [19], which can generate strongly random networks in the activity-on-thenode format, was used to construct 180 test instances using the parameter settings in Table 2. RanGen mainly uses two parameters to define the network structure: network size $(N)$ and order strength (OS). Network size $N$ specifies the number of activities in the project network. Order strength OS is
TABLE 2: Parameter settings.

\begin{tabular}{lc}
\hline Parameters & Value \\
\hline Number of activities $(|N|)$ & $10 ; 20 ; 30$ \\
Order strength $(\mathrm{OS})$ & $0.3 ; 0.5 ; 0.7$ \\
Number of modes $\left(\left|M_{i}\right|\right)$ & $4 ; 8$ \\
Most likely activity duration & Uniformly drawn from $[1,50]$ \\
Most likely smallest activity cost & Uniformly drawn from $[1,10]$ \\
Activity cost slope & Uniformly drawn from $[1,8]$ \\
$\Psi$ & Uniformly drawn from $[0.1,1]$ \\
$\theta$ & 0.5 \\
\hline
\end{tabular}

the number of precedence relations divided by the theoretical maximum number of the precedence relations in the network. Order strength describes the network density. The larger the OS value, the higher the network density. Herroelen and De Reyck [20] demonstrated that OS is better than other commonly used measures (e.g., network complexity, which is adopted in PSPLIB [21]) when describing the network topology.

Specifying 3 settings for the number of activities, 2 settings for the number of execution modes, and 3 settings for OS, we generated 10 problem instances for each of the $3 \times$ $2 \times 3$ parameter settings, resulting in 180 instances in total.

We also need to generate the most likely value of duration and cost for each activity. In DTCTP, the types of cost functions can be linear, convex, concave, or random. We study the random case, which is more general. Following Demeulemeester et al. [19], the modes of an activity were generated in the following way. First, the number of modes $\left|M_{i}\right|$ was determined according to the modes parameter listed in Table 2. Next, $\left|M_{i}\right|$ different numbers were randomly chosen from the discrete uniform distribution $[1,50]$ to form the durations that are sorted in ascending order $\left(d_{i m}, d_{i(m-1)}, \ldots, d_{i 1}\right)$. To determine the costs, starting with the normal duration mode $d_{i m}$, its corresponding cost $c_{i m}$ is randomly chosen from the discrete uniform distribution $[1,10]$. By randomly choosing a slope $s$ from the discrete uniform distribution $[1,8]$, 
we can calculate the cost of the next mode as $c_{i(m-1)}=c_{i m}+$ $s\left(d_{i m}-d_{i(m-1)}\right)$, and we repeated this stepwise procedure until the mode of maximum cost was reached.

Our model deals with uncertain data, so we generated the maximum deviation of activity duration and cost by letting $\widehat{d}_{i m}=\psi d_{i m}$ and $\widehat{c}_{i m}=\psi c_{i m}$, where $\psi \sim U[0.1,1]$. In addition, the project budget was calculated by $R=R_{\min }+\theta\left(R_{\max }-\right.$ $R_{\text {min }}$ ), where $R_{\text {min }}=\sum_{i} \min _{m}\left\{c_{i m}\right\}, R_{\max }=\sum_{i} \max _{m}\left\{c_{i m}\right\}$, and $\theta=0.5$.

4.2. Experimental Results. The parameter $\Gamma_{i}$ represents the level of robustness of our model. To study the impact of different $\Gamma_{i}$ on solutions, we set 5 different values for $\Gamma_{i}$ : $\Gamma^{(j)}=\eta_{j} /\left(|N| \times\left|M_{i}\right|\right)$, where $\eta_{j}=0,0.25,0.5,0.75,1$ and $j=0,1,2,3,4$. We see that the value of $\Gamma_{i}$ is quite small, which is because we find that even a slight deviation of $\Gamma_{i}$ has a high impact on the schedule stability (see next section). Note that our model becomes the deterministic DTCTP model when $\Gamma^{(0)}=0$. We solved the 180 problem instances for each $\Gamma_{i}$ with a CPU time limit of 300 seconds; the results are listed in Table 3. The column " $|N|$ " represents the number of activities, "OS" represents the order strength, and " $|M|$ " represents the number of modes. Columns "Avg," "Min," and "Max" provide the average, minimum, and maximum objective function values, respectively. The column "\%" shows the percentage of problems solved optimally.

The results in Table 3 demonstrate that, with the increase of order strength, the objective function value (i.e., the project makespan) increases, while the number of modes exhibits a contrary behavior. The reason for this behavior is that a higher order strength value means that more precedence relationships are involved, resulting in a longer project duration. More modes provide more choices for the durations of the activities, thus making it possible to shorten the project duration.

Small problem instances $(|N|=10)$ can be solved optimally within 300 seconds. An increase of the number of activities decreases the percentage of problem instances solved optimally. We also observed that the larger the value of $\Gamma_{i}$, the lower the percentage of problem instances solved optimally. $\Gamma_{i}$ reflects the trade-off between the robustness and the optimality of the solution. A larger $\Gamma_{i}$ results in a more stable schedule at the cost of a prolonged project duration. In addition, compared with the deterministic model $\left(\Gamma^{(0)}=0\right)$, due to the introduction of many auxiliary variables, solving the robust optimization model takes more time, thereby resulting in less optimal solutions. Accordingly, a smaller $\Gamma_{i}$ is accompanied by a lower computational requirement.

4.3. Impact of Different Factors on the Schedule Stability. Robust optimization improves the schedule robustness at the expense of prolonging the project duration. Therefore, we are interested in the trade-off between the advantages (improved schedule stability) and the disadvantages (increased project duration) of robust optimization. Specifically, from the viewpoint of stability cost, we use simulation to investigate the impact of project network structure parameters (i.e., the number of activities, the order strength, and the number of modes) on the schedule stability.
4.3.1. Simulation Design. We use the stability cost $C_{R}$ to measure the schedule stability. $C_{R}$ is defined as the weighted sum of the expected absolute difference between the planned and the actually realized activity start times [22, 23]:

$$
C_{R}=\sum_{i} w_{i} E\left(\mathbf{s}_{i}-s_{i}^{B}\right)
$$

where $s_{i}^{B}$ is the planned start time of activity $i$ in the baseline schedule, $s_{i}$ is a random variable indicating the actual start time of activity $i$, and $w_{i}$ represents the cost-per-time unit incurred by the situation that the actual start time is later than the planned time for activity $i$, with $w_{i}>0$. The value of $w_{i}$ reflects how difficult it is to change a baseline schedule or how important it is to start an activity on time. The more robust a schedule, the smaller the corresponding stability cost.

It is not realistic to use a full factorial experiment to analyze the impact of different factors on the schedule stability [24]. Instead, we focus on the main effect of different factors. When we study the impact of one factor on the average stability cost, we keep the other factors fixed at a certain level.

The simulation strategy is as follows. (1) The weights $w_{i}$ are drawn from a uniform distribution $U[1,4]$. (2) The activity mode is determined by the solutions of our proactive scheduling model. (3) The activity duration is drawn from the triangular distribution $\operatorname{TRIA}(a, m, b)$, where $a=\underline{d}_{i m}$, $m=d_{i m}$, and $b=\bar{d}_{i m}$. Note that because our model can deal with any kind of distribution, we can also draw the activity duration from other distributions. (4) We use a railway scheduling policy to determine the activity of actual start time $\mathbf{s}_{i}$. In railway scheduling, the actual start time is always later than or equal to the planned start time (as is the case with train timetable, flight scheduling, etc.). Therefore, on the condition that the precedence relationship is already satisfied, $\mathbf{s}_{i}$ is calculated according to

$$
\mathbf{s}_{i}= \begin{cases}\mathbf{s}_{i} & \text { if } \mathbf{s}_{i} \geq s_{i}^{B} \\ s_{i}^{B} & \text { otherwise }\end{cases}
$$

where $s_{i}^{B}$ is the start time of activity $i$ obtained by solving our proactive scheduling model.

For each instance in our data set, the number of simulation replications is set to 100 . Note that the stability costs mentioned in the following are always the average value.

4.3.2. Impact of the Number of Activities. Figure 2 shows the impact of the number of activities on the average schedule stability cost, while the other two factors are fixed ( $\mathrm{OS}=0.5$, $|M|=4)$. In Figure 2, the $x$-axis represents $\Gamma$. There are two $y$-axes. The $y$-axis on the left shows the stability cost and the one on the right indicates the objective function value (i.e., project makespan). For the legend in Figure 2, the letters " $\mathrm{s}$ " and "o" in brackets mean that the corresponding lines correspond to the stability cost and the objective function value, respectively. Note that since we cannot find optimal solutions for most instances with $|N|=30$ within our time limit, the results for data set $|N|=30$ will not be included in this subsection to ensure the validity of the analysis. 


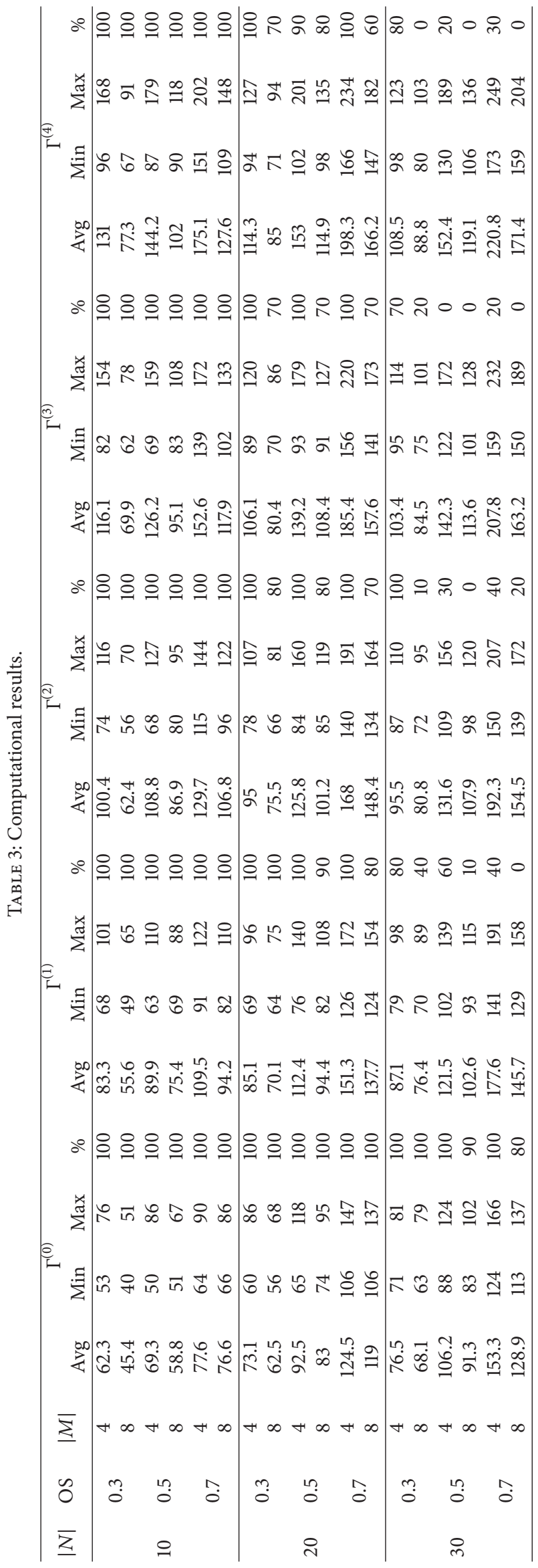




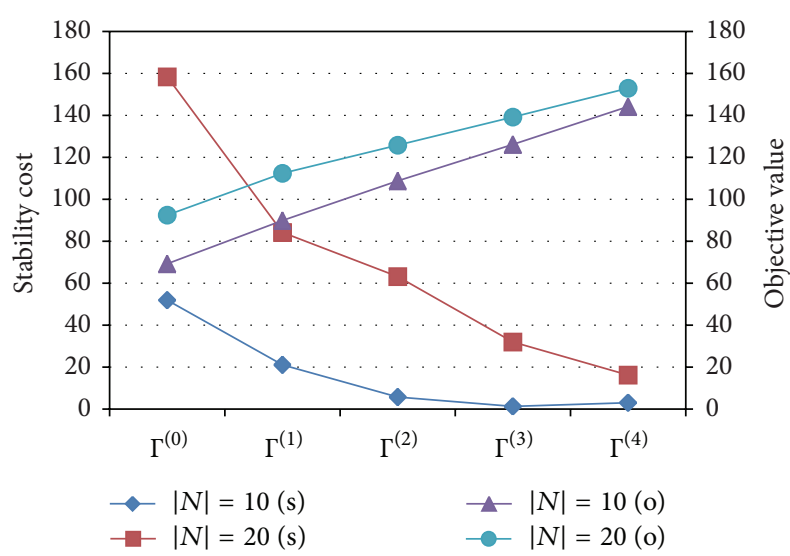

FIGURE 2: Impact of the number of activities on the schedule stability $(\mathrm{OS}=0.5,|M|=4)$.

For each robustness level $\Gamma$, with the increase in the number of activities, the stability cost increases. In other words, the larger the number of activities, the more unstable the schedule.

We see that although the actual difference between $\Gamma^{(0)}$ and $\Gamma^{(4)}$ is small, it is already sufficient to heavily reduce the stability cost when $\Gamma$ changes from $\Gamma^{(0)}$ to $\Gamma^{(4)}$. For $|N|=10$, it is interesting that the impact of $\Gamma$ on the schedule stability cost becomes very weak when $\Gamma$ is equal to or greater than $\Gamma^{(2)}$. However, the impact of $\Gamma$ on the objective function value is still positive. This means that for small scale project, it is not necessary to use a high value for $\Gamma$, as this will not reduce the schedule stability cost significantly. Instead, a high value for $\Gamma$ will prolong the project duration in this case.

4.3.3. Impact of the Order Strength. Figure 3 indicates the impact of the order strength on the average schedule stability cost, while the other two factors are fixed $(|N|=20,|M|=$ $4)$. The impact of the order strength on the stability cost is dependent on the robustness level $\Gamma$. When $\Gamma$ is low, the order strength has a positive impact on the stability cost. However, this impact becomes weak with the increase of $\Gamma$. We may conclude that when we choose a relatively high value for $\Gamma$, we could pay little attention to the density of the project precedence relations due to the weak impact of the order strength.

An interesting finding is that when $\Gamma$ increases, the magnitude of increase in the objective function value (i.e., project duration) for different order strength is basically the same. However, the magnitude of the corresponding changes in the schedule stability cost is much greater, especially when the level of the order strength is high (i.e., OS = 0.7). In other words, when the density of the project is high, the stability of a schedule can be heavily enhanced by using our proactive model and the corresponding loss in the project duration is quite small.

4.3.4. Impact of the Number of Modes. Figure 4 gives the impact of the number of modes on the average schedule stability cost, while the other two factors are fixed $(|N|=20$,

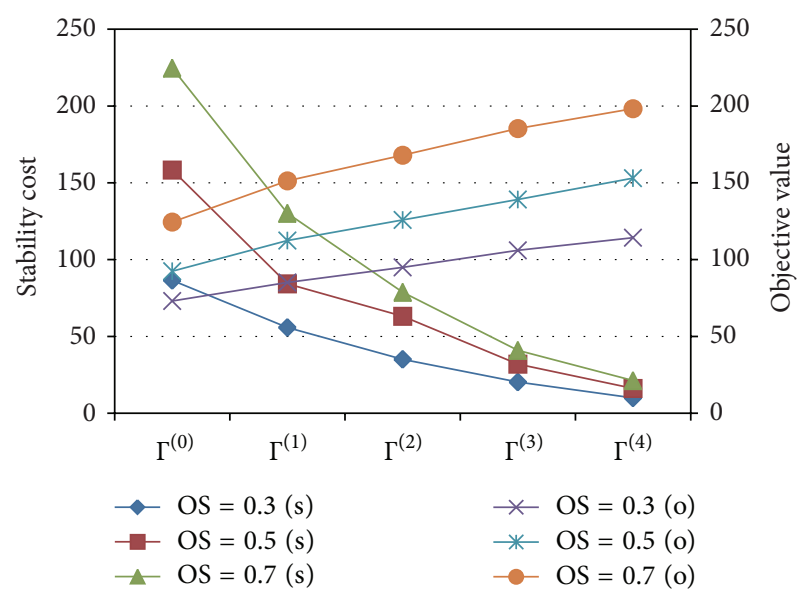

FIGURE 3: Impact of the order strength on the schedule stability $(|N|=20,|M|=4)$.

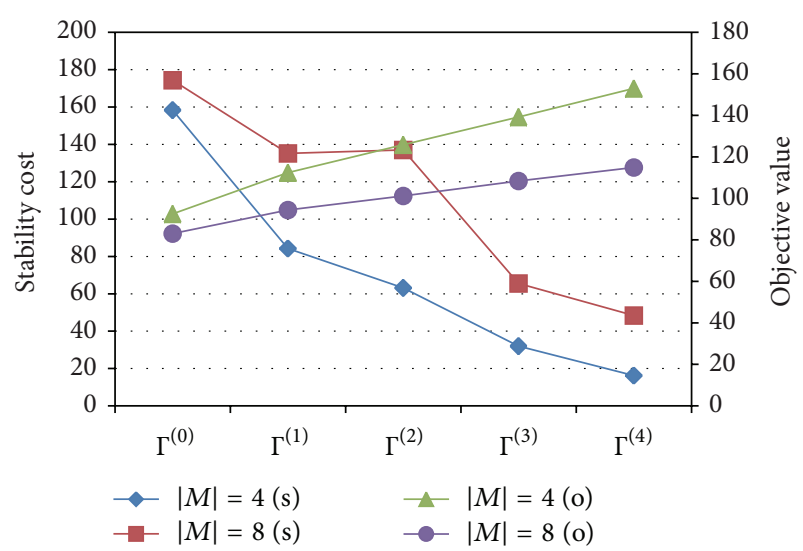

FIGURE 4: Impact of the number of modes on the schedule stability $(|N|=20$, OS $=0.5)$.

$\mathrm{OS}=0.5$ ). The results shown in Figure 4 are not uncommon. For each robustness level $\Gamma$, with the increase in the number of modes, the stability cost increases. In other words, the more modes an activity can choose, the more unstable the schedule becomes.

Moreover, in most cases, for any factor (the number of activities, the order strength, or the number of modes), Figures 2, 3, and 4 also reveal that a larger robustness level $\Gamma$ results in a smaller stability cost and this is at the cost of prolonged project duration. This further confirms our results of Section 4.2 that the greater the robustness level $\Gamma$ is, the more stable the baseline schedule is.

\section{Conclusions}

In this paper, we presented a proactive scheduling model for the project budget version of the stochastic discrete time/cost trade-off problem based on robust optimization theory. Computational experience on the randomly generated problem dataset validated our model. We also used simulation to 
analyze the impact of different project network parameters on the schedule stability.

From the experiments in the stochastic time/cost tradeoff environment, we conclude that (1) a larger value of the robustness level parameter $\Gamma$ leads to a more stable baseline schedule. However, the schedule robustness is improved at the expense of prolonging the project duration. Therefore, whether or not to adopt robust optimization depends on the decision-makers' preference. However, we recommend to use our approach when the density of the project precedence relations is high (i.e., the order strength is high). Because in this case, the loss on the project duration for obtaining a robust schedule is quite small. (2) Both the project duration and the schedule robustness are very sensitive to $\Gamma$. Even a slight increase in $\Gamma$ is already sufficient to obtain an enough robust schedule. (3) In our proactive model, the order strength has a positive impact on the project makespan, while the number of modes has a negative impact. (4) In most cases, with the increase in the number of activities, the order strength, and the number of modes, the schedule stability decreases. However, when $\Gamma$ is relatively high, the impact of the order strength on the schedule stability cost becomes weak. This means that we can ignore the factor of the order strength when we use a high value for $\Gamma$. (5) For small scale projects, it is easy to generate a quite stable schedule with a small $\Gamma$. However, for relatively large scale projects, we have to increase $\Gamma$ to make the resulting schedule robust enough.

\section{Conflict of Interests}

The authors declare that there is no conflict of interests regarding the publication of this paper.

\section{Acknowledgments}

The authors thank the reviewers for providing valuable suggestions that have improved the quality of this paper. This research was supported by the National Science Foundation of China under Grant 71271019, the Humanities and Social Sciences Foundation of the Ministry of Education under Grant 12YJA630158, and the Soft Science Research Program of Shanghai under Grant 14692105900.

\section{References}

[1] E. L. Demeulemeester and W. Herroelen, Project Scheduling: A Research Handbook, Kluwer Academic, 2002.

[2] R. T. Harvey and J. H. Patterson, "An implicit enumeration algorithm for the time/cost tradeoff problem in project network analysis," Foundations of Control Engineering, vol. 4, no. 2, pp. 107-117, 1979.

[3] T. J. Hindelang and J. F. Muth, "A dynamic programming algorithm for decision CPM networks," Operations Research, vol. 27, no. 2, pp. 225-241, 1979.

[4] P. Brucker, A. Drexl, R. Möhring, K. Neumann, and E. Pesch, "Resource-constrained project scheduling: notation, classification, models, and methods," European Journal of Operational Research, vol. 112, no. 1, pp. 3-41, 1999.
[5] F. Ballestín and R. Leus, "Resource-constrained project scheduling for timely project completion with stochastic activity durations," Production and Operations Management, vol. 18, no. 4, pp. 459-474, 2009.

[6] Ö. Hazır, E. Erel, and Y. Gnalay, "Robust optimization models for the discrete time/cost trade-off problem," International Journal of Production Economics, vol. 130, no. 1, pp. 87-95, 2011.

[7] W. J. Gutjahr, C. Strauss, and E. Wagner, "A stochastic branchand-bound approach to activity crashing in project management," INFORMS Journal on Computing, vol. 12, no. 2, pp. 125$135,2000$.

[8] Z. Laslo, "Activity time-cost tradeoffs under time and cost chance constraints," Computers and Industrial Engineering, vol. 44, no. 3, pp. 365-384, 2003.

[9] I. Cohen, B. Golany, and A. Shtub, "The stochastic time-cost tradeoff problem: a robust optimization approach," Networks, vol. 49, no. 2, pp. 175-188, 2007.

[10] H. Ke, W. Ma, and Y. Ni, "Optimization models and a GA-based algorithm for stochastic time-cost trade-off problem," Applied Mathematics and Computation, vol. 215, no. 1, pp. 308-313, 2009.

[11] E. Klerides and E. Hadjiconstantinou, "A decomposition-based stochastic programming approach for the project scheduling problem under time/cost trade-off settings and uncertain durations," Computers and Operations Research, vol. 37, no. 12, pp. 2131-2140, 2010.

[12] W. M. Ma, Q. H. Liu, and H. Ke, "A stochastic time-cost-quality trade off model for Discrete Project Scheduling problem," in Proceedings of the International Conference on Management Science and Management Innovation, Atlantis Press, 2014.

[13] W. Herroelen and R. Leus, "Robust and reactive project scheduling: a review and classification of procedures," International Journal of Production Research, vol. 42, no. 8, pp. 1599-1620, 2004.

[14] E. Demeulemeester and W. Herroelen, "Robust project scheduling," Foundations and Trends in Technology, Information and Operations Management, vol. 3, no. 3-4, pp. 201-376, 2009.

[15] P. De, E. James Dunne, J. B. Ghosh, and C. E. Wells, "The discrete time-cost tradeoff problem revisited," European Journal of Operational Research, vol. 81, no. 2, pp. 225-238, 1995.

[16] P. De, E. J. Dunne, J. B. Ghosh, and C. E. Wells, "Complexity of the discrete time-cost tradeoff problem for project networks," Operations Research, vol. 45, no. 2, pp. 302-306, 1997.

[17] D. Bertsimas and M. Sim, "Robust discrete optimization and network flows," Mathematical Programming, vol. 98, no. 1-3, pp. 49-71, 2003.

[18] D. Bertsimas and M. Sim, “The price of robustness," Operations Research, vol. 52, no. 1, pp. 35-53, 2004.

[19] E. Demeulemeester, M. Vanhoucke, and W. Herroelen, "RanGen: a random network generator for activity-on-the-node networks," Journal of Scheduling, vol. 6, no. 1, pp. 17-38, 2003.

[20] W. Herroelen and B. de Reyck, "Phase transitions in project scheduling," Journal of the Operational Research Society, vol. 50, no. 2, pp. 148-156, 1999.

[21] R. Kolisch and A. Sprecher, "PSPLIB-a project scheduling problem library: OR software-ORSEP operations research software exchange program," European Journal of Operational Research, vol. 96, no. 1, pp. 205-216, 1997.

[22] R. Leus and W. Herroelen, "Stability and resource allocation in project planning," IIE Transactions, vol. 36, no. 7, pp. 667-682, 2004. 
[23] R. Leus and W. Herroelen, "The complexity of machine scheduling for stability with a single disrupted job," Operations Research Letters, vol. 33, no. 2, pp. 151-156, 2005.

[24] S. van de Vonder, E. Demeulemeester, W. Herroelen, and R. Leus, "The use of buffers in project management: the trade-off between stability and makespan," International Journal of Production Economics, vol. 97, no. 2, pp. 227-240, 2005. 


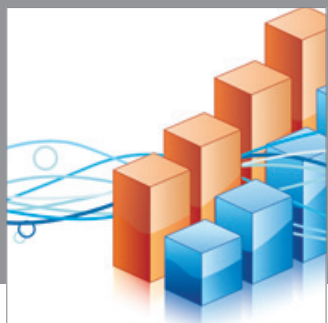

Advances in

Operations Research

mansans

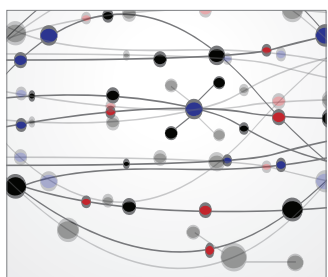

The Scientific World Journal
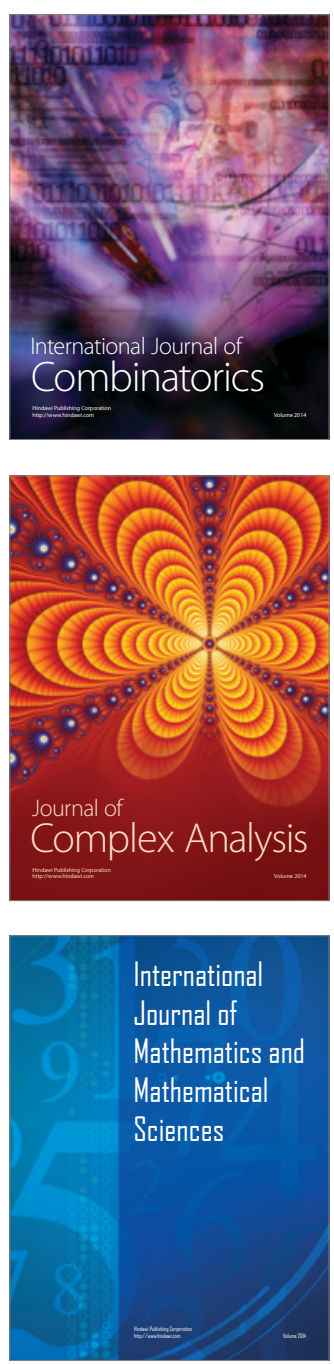
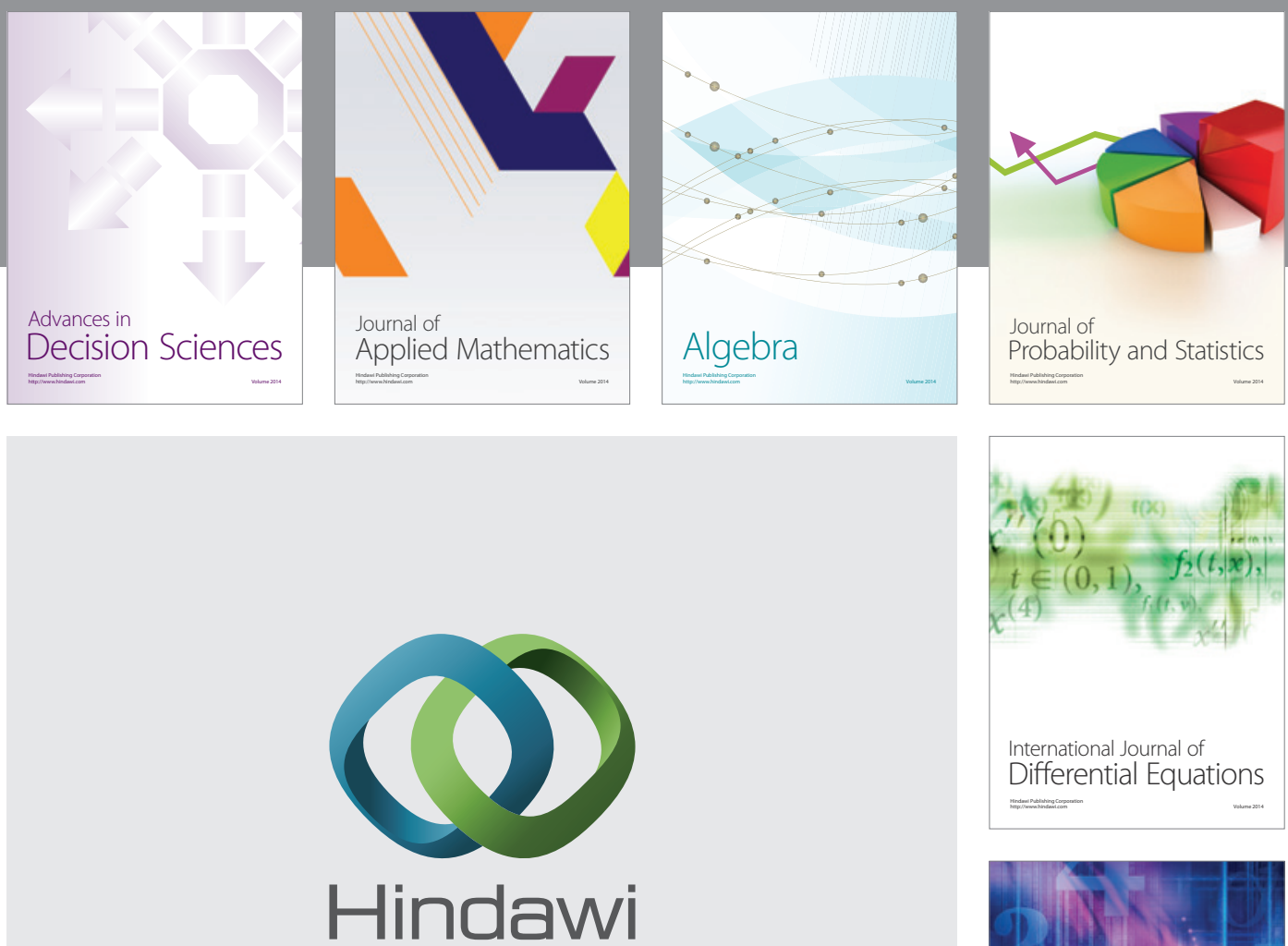

Submit your manuscripts at http://www.hindawi.com
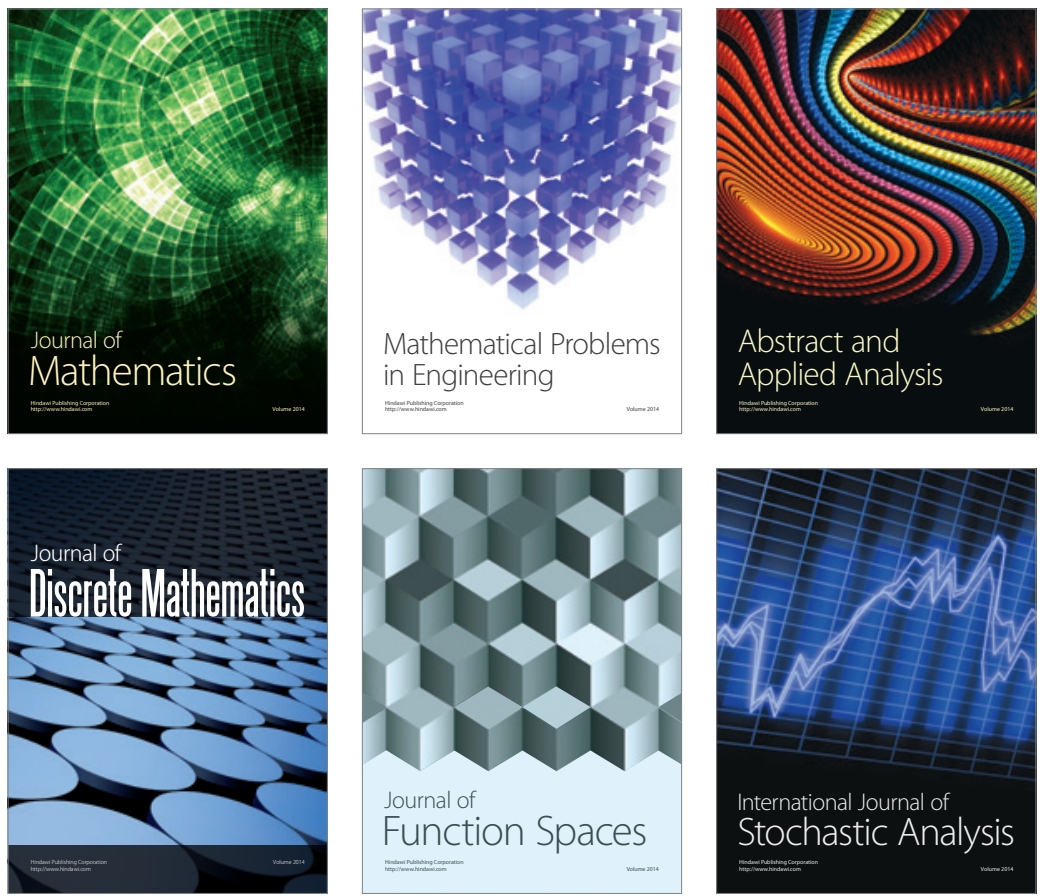

Journal of

Function Spaces

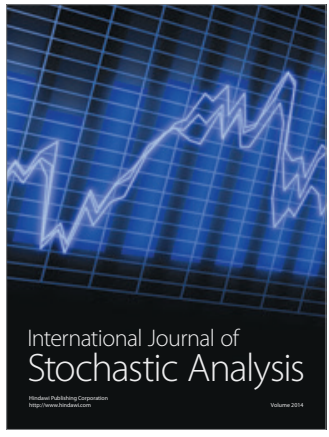

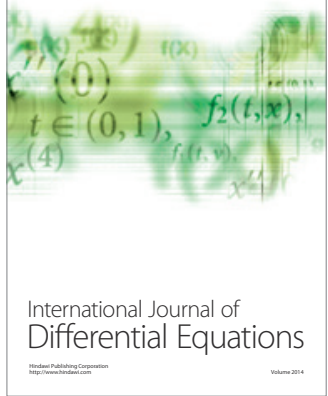
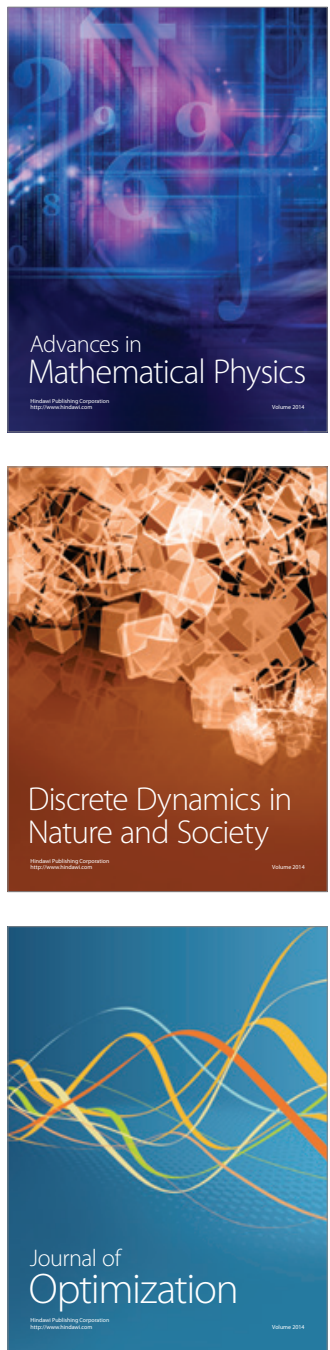